REVIEW ARTICLE

\title{
Tuberculosis: Global and Regional Scenarios
}

\author{
Md. Mahfuzar Rahman \\ Prof. Dr. Md. Mahfuzar Rahman, Professor \& Head, Department of Community Medicine \\ Anwer Khan Mordern Medical College Dhanmondi, Dhaka-1205
}

Tuberculosis is a devastating public health problem with grave socio-economic consequences. Tuberculosis causes an enormous burden of morbidity and mortality around the world. TB is infecting about a third of the global population and causing an estimated two million deaths every year. In Bangladesh a new TB case develops in every two minutes and one dies due to tuberculosis in every ten minutes. It causes a great deal of ill health in the populations of most low income countries like Bangladesh. It is also known as the disease of poverty. It is the single most common cause of death in the individuals at the reproductive age.

Tuberculosis has been a major public health problem for centuries and creating enormous burden of morbidity and mortality around the world. Yet today, despite the availability of affordable, effective treatment, the annual toll of 9 million new TB cases and 2 million TB deaths worldwide represents an intolerable burden of human suffering and unacceptable barrier to socioeconomic development. Tuberculosis is the second leading cause of death among infectious diseases worldwide ${ }^{1}$.

Tuberculosis (TB) is a very old disease, which is still a key public health challenge in Bangladesh. Tuberculosis is the leading infectious disease causing more than a quarter of the world's preventable deaths. In high burden, low income countries as in Bangladesh Tuberculosis cause more then $25 \%$ of the avoidable death. Tuberculosis is a major health problem and one of the significant causes of death in Bangladesh. According to present estimation, approximately 300,000 people fall ill of Tuberculosis and 70,000 die each year in Bangladesh. The problem is aggravated by the increasing population density, rapid urbanization, poverty and illiteracy. So, tuberculosis is also called a disease of poverty. It is an infectious disease cause by bacilli called
Mycobacterium tuberculosis. The bacilli usually enter the body by inhalation through the lung and spread to other parts of body via blood stream, the lymphatic system or through direct extension to other organs. Tuberculosis of the lung or Pulmonary Tuberculosis is the most common form of tuberculosis and occurs in about $80 \%$ of cases. It causes a great deal of ill health in the population of most low income countries and it is the single most common cause of death in individuals aged fifteen to forty nine years. It mostly affects individuals in the age of re-productivity ${ }^{2}$.

In 1993 the World Health Organization (WHO) declared Tuberculosis as a global emergency and recommended a standard strategy for control of the disease that since 1993 is known as the Directly Observed Treatment Short course (DOTS) strategy. DOTS as recommended by WHO is the most effective strategy available for controlling the TB epidemic. The NTP (National Tuberculosis Control Program) of Bangladesh follows this strategy to achieve its objectives and targets. Successful tuberculosis control depends on proper case detection by sputum smear microscopy, effective treatment with adequate drug combination, close supervision to ensure good patient compliance and monitoring the treatment. Strict adherence to treatment should be ensured to cure the patients and prevent the development of drug resistance in smear positive patients.

Directly Observed Treatment Short Course (DOTS) is very important component of the DOTS strategy. DOTS imply that an observer watches a patient swallowing the drugs, which is essential for treatment and recovery from TB. This ensures that the patient takes the right anti-TB drugs, in the right doses, at the right intervals and for the right period. All patients irrespective of category should receive all doses of anti-TB drugs under DOTS. Lack of adherence is the main cause of 
development of Multi Drug Resistant Tuberculosis (MDR-TB) $)^{2}$.

Early diagnosis of tuberculosis and effective treatment are the key elements in reduction of transmission of infection and finally achieving elimination of TB. World Health Organization (WHO) has set the international target value for a favorable treatment outcome at $85 \%$. Incomplete treatment carries a risk of development of resistance, increased disease transmission, and increased morbidity and mortality ${ }^{3}$.

TB usually affects the lungs, but it can also affect other parts of the body, such as the brain, the kidneys or the spine ${ }^{4}$. Persons with latent TB infection are not infectious and cannot spread TB infection to others. However, persons with active TB disease usually have symptoms, are considered infectious, and may spread TB bacteria to others. The general symptoms of TB disease include feelings of sickness or weakness, weight loss, fever, and night sweats. The symptoms of TB disease of lungs may also include coughing, chest pain, and the coughing up of blood. Symptoms of TB disease in other parts of the body depend on the area affected. People with latent TB infection can not spread TB to others; nothing further will need to be done in the work place. However, in active TB disease, the TB control program may start a contact investigation to detect source as well as who else might be at risk ${ }^{4}$.

In Bangladesh district hospitals and some infectious disease and specialized hospitals constituted the second level of referral for health care. There were 12 tuberculosis hospitals (20 to 120 beds each) and forty-four tuberculosis clinics ${ }^{6}$. It was found that private practitioners including chest physicians are often reluctant to having a successful National Tuberculosis Program (NTP) in low and middle income countries. Because of their tendency to treat the patient for a long period of time and through and that the patient visits him frequents for his monetary gain. This complicated situation has been present in most of the Latin American countries for many years ${ }^{7}$.

There are concerns that the NTP devotes insufficient attention to smear negative cases and that it has barriers to care for the homeless and for those who refuse supervision therapy. There is need to understand and take continually into account people's perceptions, health-seeking behavior and expectations from the healthcare services ${ }^{8}$.

Most of the disease transmission in TB takes place before the diagnosis of TB is made and treatment started. Delay in TB diagnosis therefore leads to an increase in disease transmission. Several studies have sought to establish whether the delay in diagnosis is due to a delay in seeking care or due to inability of the provider to diagnose promptly. Commonly, the delay is in receiving a diagnosis rather than in seeking care ${ }^{8}$. A study of TB patients and practitioners in private clinics in India showed median delays in diagnosis of about 3 weeks and 2 weeks respectively among urban and rural patients after they sought help at private clinics. About $33 \%$ of urban patients and $36 \%$ of the rural patients had not been diagnosed even after 4 weeks of seeking help ${ }^{8}$.

Moreover, study conducted in India also identifies and highlights the need to educate private practitioners and TB patients, and indicate ways of meaningful involvement in efforts to revitalize the national TB control program ${ }^{9}$. Another study also reveals gaps and weakness in the private doctors reported practice of managing lung tuberculosis, the most important and persistent problem of public health concern in India ${ }^{10}$. It was also found that the Private Practitioners (PPs) are not aware of or are not prescribing, the treatment regimen recommended by the Revised NTP, and majority of the patients are being over-treated. PPs need more training, and more collaborative efforts are required between public health facilities and practicing doctors ${ }^{11}$. However, study indicates many misunderstandings in the field of transmission, BCG vaccination and the performance of the NTP among general practitioners. Sputum examinations were considerably neglected in case findings / diagnosis and treatment monitoring. As for treatment, $89 \%$ were giving either non-recommendable regimens or bad regimens ${ }^{12}$. Nevertheless, study emphasizes collaborative efforts between private practitioners and the government can achieve moderate high rates of case detection and high rates of treatment success ${ }^{13}$. 
Private practitioners (PPs) are generally the first point of contact for significant proportion of patients with tuberculosis. All PPs can support and encourage effective tuberculosis control by ensuring prompt referral, providing reassurance, giving only recommended revised NTCP drug regimens ${ }^{14}$. In Sri Lanka the national tuberculosis program had briefed PPs on DOTS and they are now referring the patients to public sector. However, many private practitioners continue to treat $\mathrm{TB}$ patients without reporting them to the national program. Thailand has introduced innovative approaches to train and inform the informal sector including drug vendors and traditional healers to increase community awareness to prevent self-medication ${ }^{15}$.

Directly Observed Treatment Short course (DOTS) is very important strategy for providing anti $\mathrm{TB}$ treatment to sputum positive TB cases and has already been proved with a high cure rate. DOTS strategy is designed to enhance strict adherence to anti-TB treatment as well as to increase treatment success rate. DOTS have achieved about $80 \%$ treatment success rate and $95 \%$ coverage in 2000 in Bangladesh. Still non compliant TB patients are creating potential threat for spreading MDR-TB in our country. With proper treatment, almost all cases of TB are curable. But proper treatment is not always easy to attain. However, because of the drugs often cause unpleasant side effects and because patients start feeling better after few months, not everyone completes the full course of treatment. TB bacteria that linger following incomplete therapy are likely to resist antituberculosis drugs in future and flare-ups. Worse still, people with active cases of MDR-TB can pass those super bugs on to new victims. So, unfortunately, partial treatment for $\mathrm{TB}$ is worse than no treatment at all.

DOTS expanded rapidly in the South-East Asian Region over the period of the Partnership's first Global Plan (2001-2005), and 100\% geographical coverage was achieved in 2005. All the Region's TB high-burden countries (Bangladesh, India, Indonesia, Myanmar and Thailand) have made impressive progress in improving coverage and quality. Case detection increased from a mere $18 \%$ in 2000 to $45 \%$ in 2003 and was expected to reach about $65 \%$ by the end of 2005, against the World Health Assembly and Stop TB Partnership's 2005 target of $70 \%$. The treatment success rate in the region is already $85.3 \%$, meeting the 2005 target of $85 \%$. This progress has been made possible through strong political commitment and large investments in improved infrastructure, reliable drug supply, increased staffing, improved laboratory services, and intensified training and supervision. Coverage of drug resistance surveillance is low in the Region, mainly because of limited data from Bangladesh, India and Indonesia, making it difficult to assess the regional MDR-TB situation. Available data show that, while the levels of MDR-TB among previously untreated cases may be below 3\%, the large numbers of TB cases translate into a significant burden of MDR-TB in South-East Asia. It is estimated that $25 \%$ of all MDR-TB cases worldwide are in India alone ${ }^{1}$.

DOTS have been applied for the new and re treatment sputum positive pulmonary tuberculosis cases. Under DOTS patient have to take anti TB drugs in front of drug provider on daily basis. DOTS is a strategy that provides the most effective medicines to tuberculosis patient, ensures that they regularly take those medicines until they are cured. DOTS provide the safest, most powerful knockout punch against tuberculosis in the shortest period of time. DOTS produce cure rates as high as 95 percent; DOTS stops tuberculosis bacteria at the source; DOTS prevents multi drug resistant tuberculosis form developing. DOTS is the cost effective approach for stopping tuberculosis transmission. Lack of strict adherence to DOTS is causing re-emergence of TB as well as MDRTB/XDR-TB worldwide. Present estimate shows that $3 \%$ of the newly diagnosed cases are MDR-TB and of them $19 \%$ have the history of non adherence to DOTS $^{16}$.

Multidrug-resistant tuberculosis (MDR TB) is a form of tuberculosis that is resistant to two or more of the primary drugs (isoniazied and rifampin) used for the treatment of tuberculosis. Extensively drugresistant $\mathrm{TB}$ (XDR TB) is TB resistant to at least isoniazied and rifampin among the first-line anitTB drugs and among second-line drugs, is resistant to any fluoroquinolone and at least one of three injectable drugs. Resistance to one or several 
forms of treatment occurs when the bacteria develops the ability to withstand antibiotic attack and relay that ability to newly produced bacteria. Since that entire strain of bacteria inherits this capacity to resist the effects of the various treatments, resistance can spread from one person to another. On an individual basis, however, inadequate treatment or improper use of the antituberculosis medications remains an important cause of drug-resistant tuberculosis. Drug-resistant TB is difficult and costly to treat and can be fata ${ }^{17}$.

The World Health Organization estimates that up to 50 million persons worldwide may be infected with drug resistant strains of TB. Also, 300,000 new cases of MDR-TB are diagnosed around the world each year and 79 percent of the MDR-TB cases now show resistance to three or more drugs. A strain of MDR TB originally develops when a case of drug-susceptible tuberculosis is improperly or incompletely treated. This occurs when a physician does not prescribe proper treatment regimens or when a patient is unable to adhere to therapy. Improper treatment allows individual TB bacilli that have natural resistance to a drug to multiply. Eventually the majority of bacilli in the body are resistant. Once a strain of MDR TB develops it can be transmitted to others just like a normal drug-susceptible strain. MDR-TB is more difficult to treat than drug-susceptible strains of TB. The success of treatment depends upon how quickly a case of TB is identified as drug resistant and whether an effective drug therapy is available. The second-line drugs used in cases of MDR-TB are often less effective and more likely to cause side effects $^{18}$.

India, Indonesia and Bangladesh, three of the most populous countries were major contributors of TB cases. Five countries, namely Bangladesh, India, Indonesia, Myanmar and Thailand account for more than $95 \%$ of the cases in the region. Tuberculosis is the biggest killer among adults in the region. Tuberculin positivity is more than $50 \%$ of adults in Bangladesh is indicative of high incidence of active disease in the country ${ }^{19}$.

\section{References}

1. Global plan to stop TB, 2006-2015. Part II: Global and regional scenarios for TB control 2006-2015

2. Tuberculosis and Leprosy Control Services. National Guidelines and Operational Manual for Tuberculosis Control (3rd Editions): Director General of Health Services, Bangladesh; July-2006.

3. Vasankari T, Pekka H, Ollgren J, Kari L, Maarit K, Petri $\mathrm{R}$, et al. Risk factors for poor tuberculosis treatment outcome in Finland. a cohort study, Department of Infectious Disease Epidemiology, National Public Health Institute, 166 Helsinki, Finland BMC Public Health 2007.

4. www.cdc.gov/tb/pubs/tbfactsheets/nonhealthcare employers.htm.

5. www.searo.who.int/en/section10/ection2097/section2098 10633.htm

6. http://countrystudies.us/bangladesh/50.htm

7. http://www.sciencedirect.com/science?

8. $\mathrm{WHO} / \mathrm{CD} / \mathrm{TB} / 2001.285$ Involving Private Practitioners in Tuberculosis Control: Issues, interventions, and emerging policy framework

9. http://www.ncbi.nlm.nih.gov/sites/entrez? Db

10. http://www.ncbi.nlm.nih.gov/sites/entrez?Db

11. http://www.ncbi.nlm.nih.gov/sites/entrez?cmd

12. http://www.ncbi.nlm.nih.gov/sites/entrez?Db

13. http://www.ncbi.nlm.nih.gov/sites/entrez?Db

14. Involvement of Private Practitioners in the revised National Tuberculosis Control program; Central TB Division, Directorate General of health Services, Ministry of Health \& Family Welfare, Nirman Bhavan, New Delhi-110 011.

15. http://searo.who.int/en/section10/section $18 /$ section $356 /$ se ction413 1889.htm.

16. A N Zafar Ullah, James N Newell, Jalal Uddin Ahmed, M K A Hyder, Akramul Islam. Government-NGO collaboration the case of tuberculosis control in Bangladesh; January-2006.

17. American lung association. Multi drug-Resistant Tuberculosis Fact Sheet Home: Regional Report; April2007.

18. Tuberculosis Epidemiology in South-East Asia; WHO Regional Office for South-East Asia: 11 March 2008.

19. Rashid KM, Rahman M, Hyder S. Text Book of Community Medicine and Public Health; $4^{\text {th }}$ Edition, RHM Publishers 2004. 